\title{
Acid Stress Triggers Resistance to Acetic Acid-Induced Regulated Cell Death through Hog1 Activation Which Requires RTG2 in Yeast
}

\author{
Nicoletta Guaragnella $\left(\mathbb{D},{ }^{1,2}\right.$ Mariarita Stirpe $\mathbb{D}^{3},{ }^{3}$ Domenico Marzulli, ${ }^{1}$ Cristina Mazzoni ${ }^{3},{ }^{3}$ \\ and Sergio Giannattasio ${ }^{1}$ \\ ${ }^{1}$ Institute of Biomembranes, Bioenergetics and Molecular Biotechnologies, CNR, Via Amendola 165/a, Bari, Italy \\ ${ }^{2}$ Department of Biosciences, Biotechnology and Biopharmaceutics, University of Bari "A. Moro", Bari, Italy \\ ${ }^{3}$ Pasteur Institute-Cenci Bolognetti Foundation, Department of Biology and Biotechnology 'Charles Darwin', Sapienza University \\ of Rome, Piazzale Aldo Moro 5, 00185 Rome, Italy
}

Correspondence should be addressed to Nicoletta Guaragnella; n.guaragnella@ibiom.cnr.it and Sergio Giannattasio; s.giannattasio@ibiom.cnr.it

Received 27 July 2018; Accepted 13 December 2018; Published 25 February 2019

Academic Editor: Ralf Braun

Copyright (C) 2019 Nicoletta Guaragnella et al. This is an open access article distributed under the Creative Commons Attribution License, which permits unrestricted use, distribution, and reproduction in any medium, provided the original work is properly cited.

Acid stress causes resistance to acetic acid-induced regulated cell death (AA-RCD) in budding yeast, resulting in catalase activation. In order to explore the molecular determinants of evasion of AA-RCD triggered by acid stress adaptation, we studied the involvement and the possible interplay of the master regulator of transcription high-osmolarity glycerol 1 (HOG1) and RTG2, a positive regulator of the RTG-dependent mitochondrial retrograde signaling. Viability, DNA fragmentation, and ROS accumulation have been analyzed in wild-type and mutant cells lacking HOG1 and/or RTG2. Catalase activity and transcription of CTT1 and CTA1, coding the cytosolic and peroxisomal/mitochondrial catalase, respectively, as well as Hog1 phosphorylation, were also analyzed. Our results show that HOG1 is essential for resistance to AA-RCD and its activation results in the upregulation of CTT1, but not CTA1, transcription during acid stress adaptation. RTG2 is required for Hog1-dependent CTT1 upregulation upon acid stress, despite failure of $R T G$ pathway activation. We give evidence that $R \operatorname{tg} 2$ has a cytoprotective role and can act as a general cell stress sensor independent of Rtg1/3-dependent transcription.

\section{Introduction}

In multicellular organisms, the controlled suicide of single cells is crucial for development and homeostasis, providing a system that eliminates superfluous cells that might compromise organismal fitness. Similarly, the cellular suicide of a unicellular organism like yeast under certain circumstances provides a system to eliminate cells promoting the survival of the population as a whole. Thus, it is important to understand the mechanisms by which cells activate death or survival pathways in response to environmental changes $[1,2]$.

Various stress types compromising cell homeostasis elicit the activation of specific adaptive stress response through which extracellular information is converted into rewiring of gene expression aimed at maximizing cell survival [3].
On the other hand, cells of both multicellular and unicellular organisms can succumb through a regulated cell death (RCD) program under extreme conditions [4]. The mechanism by which yeast undergoes RCD in response to acetic acid (AA-RCD) has been investigated in details. Actively dividing yeast cells grown in glucose when shifted to media acidified to $\mathrm{pH} 3.00$ with a strong acid $(\mathrm{HCl})$ and containing $80 \mathrm{mM}$ acetic acid in the undissociated state undergo AA-RCD through a conserved mitochondrial pathway that is characterized by early ROS accumulation, cytochrome $c$ release, and mitochondrial dysfunction, as in mammalian intrinsic apoptosis [5]. We have shown that cell incubation at $\mathrm{pH} 3.00$ (acid stress) for at least twenty minutes before adding acetic acid makes yeast adapted to acetic acid stress and fully resistant to AA-RCD [6]. 
Acid-stressed yeast cells evade AA-RCD due to a specific increase in catalase activity and decrease in ROS accumulation [6, 7]. Moreover, overexpression of CTT1, encoding cytosolic catalase T, completely inhibits AA-RCD occurrence [8]. Catalase $\mathrm{T}$ expression is upregulated under different stressful conditions, including acid stress [9, 10]. The stress-activated protein kinase (SAPK) high-osmolarity glycerol 1 (Hog1) regulates CTT1 transcription through the transcription factors Msn2/Msn4 [10, 11]. Importantly, Hog 1 has been linked to acetic acid stress adaptation being responsible for the phosphorylation and subsequent degradation of aquaglyceroporin Fps1, required for cellular accumulation of acetic acid at low $\mathrm{pH}[12,13]$.

Yeast mitochondrial retrograde (RTG) signaling is a mitochondria-to-nucleus communication pathway that affects the transcription of nuclear-encoded mitochondrial genes to compensate for mitochondrial dysfunction, thereby restoring metabolic fitness. Rtg2, the positive regulator of the RTG signaling, acts as a sensor of mitochondrial dysfunction regulating the nuclear localization of the heterodimeric transcription factor Rtg1/3, which controls the transcription of RTG target genes [14]. RTG pathway activation has been shown to contribute to AA-RCD evasion under glucose derepressing conditions, whereby mitochondrial respiration increased [15]. Interestingly, SAPK Hog1 has proved to control Rtg1/3 nuclear accumulation and to regulate its binding to chromatin and transcriptional activity in response to osmostress [16].

The aim of this work was to study the role and the possible interplay of HOG and RTG-dependent signaling in AA-RCD evasion of acid-stressed yeast cells. We demonstrated that both HOG1 and RTG2 contribute to RCD evasion by protecting cells from oxidative stress and mitochondrial dysfunction in response to acetic acid treatment. The expression of CTT1, but not CTA1, encoding peroxisomal catalase $\mathrm{A}$, is upregulated by acid stress in an Rtg2-dependent manner. Finally, our results indicate that Hog1 phosphorylation is delayed in the absence of Rtg2, indicating for the first time a role of RTG signaling in Hog1 activation.

\section{Materials and Methods}

We followed the methods of Guaragnella et al. [15].

2.1. Yeast Strains, Growth Conditions, and Acetic Acid Treatment. The $S$. cerevisiae strains used in this study were W303-1B (WT) cells (MAT $\alpha$ ade2 leu2 his3 trp1 ura3), $\Delta r t g 2$ (rtg2 $\triangle:: L E U 2)$, and $\Delta$ hog1 (hog1 $:: N A T \# 2)$, which was kindly provided by Prof. Posas, Universitat Pompeu Fabra, Barcelona, Spain. The double mutant $\Delta r \operatorname{tg} 2 \Delta h o g 1$ was constructed by replacing RTG2 with the LEU2 gene ( $r$ tg2 $\Delta::$ LEU2 hog1 $\Delta::$ NAT\#2).

Cells were grown at $30^{\circ} \mathrm{C}$ in YPD ( $1 \%$ yeast extract, $2 \%$ Bacto peptone, and 2\% glucose). Acetic acid treatment was carried out as described in [4]. Briefly, cells were grown at $26^{\circ} \mathrm{C}$ up to exponential phase $\left(\mathrm{OD}_{600}=0.6-0.8\right)$ in $\mathrm{YPD}$, resuspended $\left(10^{7}\right.$ cells $\left./ \mathrm{ml}\right)$ in the same medium adjusted to pH 3.00 with $\mathrm{HCl}$, containing or not (control) $80 \mathrm{mM}$ acetic acid, and incubated for different times at $26^{\circ} \mathrm{C}$. Acid-stressed cells were maintained in YPD pH 3.0 medium for 30 minutes before addition of acetic acid. Cell viability was determined by measuring colony-forming units (CFU) after 2 days of growth on YPD plates at $30^{\circ} \mathrm{C}$.

2.2. TUNEL Assay, Intracellular ROS Detection, and Respiratory Competence Index. DNA fragmentation was detected by TUNEL assay. Acetic acid-treated and control cells $\left(2 \times 10^{7}\right)$ were harvested at $150 \mathrm{~min}$. Briefly, cells were fixed in a $3.7 \%$ formaldehyde solution in PBS, digested with $750 \mu \mathrm{g} / \mathrm{ml}$ zymolyase $20 \mathrm{~T}$, and incubated in a permeabilization solution ( $0.1 \%$ Triton X-100, $0.1 \%$ sodium citrate) for $2 \mathrm{~min}$ on ice and then with a $30 \mu \mathrm{l}$ TUNEL reaction mixture (In Situ Cell Death Detection Kit, Fluorescein, Roche) for 1 hour at $37^{\circ} \mathrm{C}$. After incubation, cells were washed, resuspended in PBS, and observed using a Leica TCS SP5 confocal microscope. To detect intracellular $\mathrm{H}_{2} \mathrm{O}_{2}, 10 \mu \mathrm{g} / \mathrm{ml}$ 2,7dihydrodichlorofluorescein diacetate $\left(\mathrm{H}_{2} \mathrm{DCF}-\mathrm{DA}\right.$, Molecular Probes) dissolved in ethanol was added to cells either $30 \mathrm{~min}$ before or during cell treatment with or without acetic acid. $2 \times 10^{7}$ acetic acid-treated cells were harvested at $30 \mathrm{~min}$, and oxidation to the fluorophore dichlorofluorescein (DCF) was detected by confocal fluorescence microscopy analysis. Respiratory competence was assessed by measuring cell capacity to grow on nonfermentable and fermentable carbon sources. The same number of cells from acetic acid-treated samples was plated on YPGlycerol (YPG) and YPD, and CFU were counted after 2-3 days. The index of respiratory competence (IRC) was defined as the percentage of cells able to grow in both YPD and YPG [17] and reported as \% of acid-stressed WT-adapted cells.

2.3. Catalase Activity Assay. Exponentially grown cells $\left(10^{8}\right)$, directly exposed to acetic acid or pretreated in acidic medium before the exposure, were sedimented by centrifugation, washed once with $50 \mathrm{mM}$ potassium phosphate buffer $\mathrm{pH}$ 7.0 , and resuspended in $0.3 \mathrm{ml}$ of the same buffer. Cells were broken through mechanical disruption of cell walls with glass beads $(0.5 \mathrm{~mm}$ BioSpec Products, Bartlesville, OK, USA) by TissueLyser by vortexing eight times for $30 \mathrm{sec}$ with $30 \mathrm{sec}$ intervals in an ice bath and centrifuged for $10 \mathrm{~min}$ $(15,000 \times g)$ to clarify the supernatant. A $20-60 \mu$ l supernatant was used for enzyme assay. All reagents used for catalytic activity measurements were from Sigma-Aldrich (St. Louis, MO, USA). The total catalase activity was measured spectrophotometrically by monitoring the disappearance of hydrogen peroxide at $240 \mathrm{~nm}$ [18]. Protein concentration was determined using the Bio-Rad protein assay (Bio-Rad Laboratories, Hercules, CA), with bovine serum albumin as a standard.

2.4. Real-Time Polymerase Chain Reaction (PCR). The mRNA levels of peroxisomal citrate synthase and peroxisomal and cytosolic catalase-encoding genes (CIT2, CTA1, and CTT1) were determined in exponentially growing cells $\left(\mathrm{OD}_{600}=0.7\right)$, after a low $\mathrm{pH}$ shift and during acetic acid treatment. $20 \mathrm{ml}$ of cell suspension was centrifuged at $3000 \times g$. Cell pellets were either stored at $-80^{\circ} \mathrm{C}$ or 
immediately used to extract total RNA with Presto Mini RNA Yeast Kit (Geneaid, Taiwan) through mechanical disruption of cell walls with glass beads by TissueLyser (Qiagen). $1 \mu \mathrm{g}$ RNA $\left(\mathrm{OD}_{260} / \mathrm{OD}_{280} \geq 1.9\right)$ reverse transcription was immediately performed using QuantiTect Reverse Transcription Kit (Qiagen), and cDNA was directly used for real-time PCR analysis or stored at $-20^{\circ} \mathrm{C}$. Real-time PCR was carried out by QuantiTect SYBR Green PCR Kit (Qiagen) on a QuantStudio 6 Flex instrument from Applied Biosystems using the following primer pairs: for CIT2: $(\mathrm{F})$ $5^{\prime}$-CGGTTATGGTCATGCTGTGCT- $3^{\prime}$ and (R) $5^{\prime}$-GGTC CATGGCAAACTTACGCT-3'; for CTA1: (F) $5^{\prime}$-CAAGCA AGAAATCTCTACCGCG- $3^{\prime}$ and (R) $5^{\prime}$-GCGCTGCTGTA TTTGAGGACA-3'; for CTT1: (F) $5^{\prime}$-GAGAAAGAGTT CCGGAGCGTGT-3' and (R) $5^{\prime}$-ATTCTGGTATGGAGCG GCGTA-3'; and for ACT1: (F) $5^{\prime}$-CTTTGGCTCCATCTTC CATG- $3^{\prime}$ and (R) $5^{\prime}$-CACCAATCCAGACGGAGTACTT$3 '$. The amount of CIT2, CTA1, and CTT1 mRNA normalized with ACT1 mRNA was calculated in arbitrary units (a.u.) using the standard curve method.

2.5. Immunoblot Analysis. Samples of total proteins were extracted according to the TCA method previously described [19], separated by electrophoresis on a denaturing gel, and transferred onto a nitrocellulose filter. After the transfer, the membrane was stained with a Ponceau S solution (Sigma-Aldrich) before immunoblotting analysis. Antiphospho-p38MAP kinase (Thr180/Tyr182) (\#9211, Cell Signaling Technology) and Hog1 (y-215) (sc-9079, Santa Cruz Biotechnology, CA, USA) antibodies (1:1000 dilutions) were used to detect phosphorylated Hoglp. Immunodetection was performed using enhanced chemiluminescence (ECL; SuperSignal system, Pierce) detected with an X-ray film (Kodak).

2.6. Statistical Analysis. Statistical analysis was performed by using paired Student's $t$-test (Excel software).

\section{Results}

3.1. AA-RCD Evasion due to Acid Stress Depends on HOG1 and RTG2. To investigate the role of HOG1 and RTG pathways in yeast AA-RCD evasion, acid-stressed WT and knockout cells lacking either HOG1 or RTG2 or both genes were compared with respect to cell sensitivity to acetic acid. As a control, WT cells were treated with acetic acid without acid stress adaptation. We found that acid-stressed $\Delta h \circ g 1$ cells progressively lose viability which decreased to about $20 \%$ at $200 \mathrm{~min}$ as for control WT cells that undergo AA-RCD, whereas acid-stressed WT cells remained fully viable, as reported in [6] (Figure 1(a)). Acid-stressed $\Delta r t g 2$ cells showed $50 \%$ viability after $200 \mathrm{~min}$ whereas $\Delta h \circ g 1 \Delta r t g 2$ behaved similarly to $\Delta$ hogl cells (Figure $1(\mathrm{a})$ ). The specific death rates of acid-stressed $\Delta$ hog 1 and $\Delta h \circ g 1 \Delta r t g 2$ cells $\left(0.015 \mathrm{~min}^{-1}\right)$ were similar to the ones measured in WT cells undergoing AA-RCD.

In order to assess the nature of cell death, DNA fragmentation was analyzed in acid-stressed cells, both with and without (control) acetic acid treatment. About $90 \%$ of $\Delta$ hog 1 and
$\Delta h o g 1 \Delta r t g 2$ cells treated with acetic acid were positive to TUNEL assay at $150 \mathrm{~min}$ (Figure 1(b)). This percentage was similar to the one measured in dying WT cells. Differently, less than $20 \%$ of acid-stressed WT cells showed DNA fragmentation. In $\Delta r t g 2$ cells, the percentage of DNA fragmentation was more than $50 \%$ at 150 min (Figure 1(b)). In the absence of acetic acid treatment, less than $5 \%$ of cells were TUNEL positive in all cell types.

Since early ROS accumulation is a typical marker of yeast AA-RCD [5], intracellular hydrogen peroxide levels were measured in WT and mutant cells at $30 \mathrm{~min}$. In $\Delta$ hog 1 and $\Delta$ hog $1 \Delta r \operatorname{tg} 2$ cells, the percentage of DCF-positive cells increased from less than $10 \%$ in the control to about $40 \%$ following acetic acid treatment (Figure 1(c)). Less than 5\% acid-stressed WT cells, which evade AA-RCD, accumulated hydrogen peroxide. Interestingly, in $\Delta r t g 2$ cells, ROS accumulation was significantly higher than in acid-stressed WT-adapted cells (20\% of positivity to DCF versus $4 \%$ measured in WT cells) but still lower than $\Delta h \circ g 1$ and $\Delta h \circ g 1 \Delta r t g 2$ (Figure 1(c)).

Mitochondrial dysfunction is another hallmark of AARCD [20]. Thus, to assess whether the loss of mitochondrial function occurred in the death-sensitive mutants, the index of respiratory competence (IRC) was evaluated as a function of time in acid-stressed $\Delta h \circ g 1, \Delta r \operatorname{tg} 2$, and $\Delta h o g 1 \Delta r t g 2$ cells treated with acetic acid. A reduction of IRC was observed in all cases, with a more drastic decline in functionality for $\Delta h o g 1$ and $\Delta h o g 1 \Delta r t g 2$ mutants with respect to $\Delta r t g 2$ cells, about $80 \%$ versus $60 \%$, respectively, compared to WT-adapted cells after $150 \mathrm{~min}$ (Figure 1(d)).

Overall, these results show that HOG1 and partially RTG signaling contribute to AA-RCD evasion due to acid stress.

3.2. RTG2 Is Required for the Full Activation of Catalase $T$ in Acid-Stressed Cells Resistant to AA-RCD. Catalase activity, almost undetectable in exponentially growing WT cells, is specifically triggered by extracellular acid stress reaching its maximum after $30 \mathrm{~min}$ and remaining high in cells protected from AA-RCD. Thus, it represents a hallmark of cells evading AA-RCD [6]. Since Hog1 is involved in the osmotic regulation of CTT1 transcription [10], catalase activity was measured in WT and knockout cells either in normal growth conditions without acetic acid (untreated) or at $30 \mathrm{~min}$ of acid stress before and after acetic acid addition (Figure 2(a)). As expected, catalase activity was high in WT cells at low $\mathrm{pH}$ and remained higher than in untreated cells after the addition of acetic acid. All mutant cells exhibited an overall significant reduction of catalase activity. However, although catalase activity showed a slight but significant increase in acid-stressed $\Delta r t g 2$ cells with and without acetic acid, it remained virtually constant in $\Delta h \circ g 1$ and $\Delta h \circ g 1 \Delta r t g 2$ cells in all conditions. These data suggest that RTG2 participates in the HOG1-dependent catalase activity increase caused by acid stress. This is in agreement with the partial and full sensitivity to AA-RCD of $r \operatorname{tg} 2$ and hog1 mutants, respectively.

The impairment of the RTG pathway and/or HOG1 deletion partially or fully restored, respectively, AA-RCD in acid-stressed cells with concomitant early accumulation of 


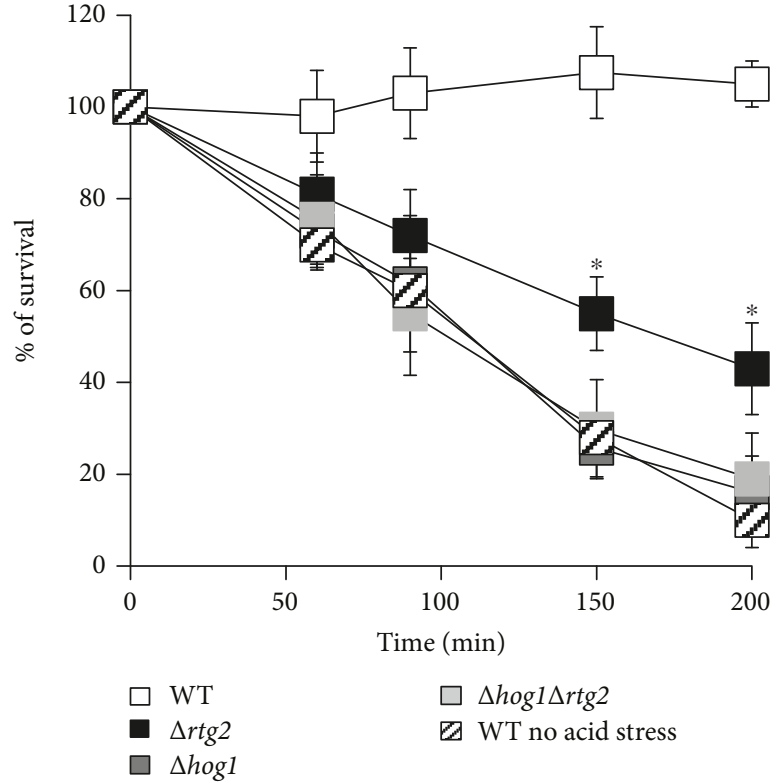

(a)

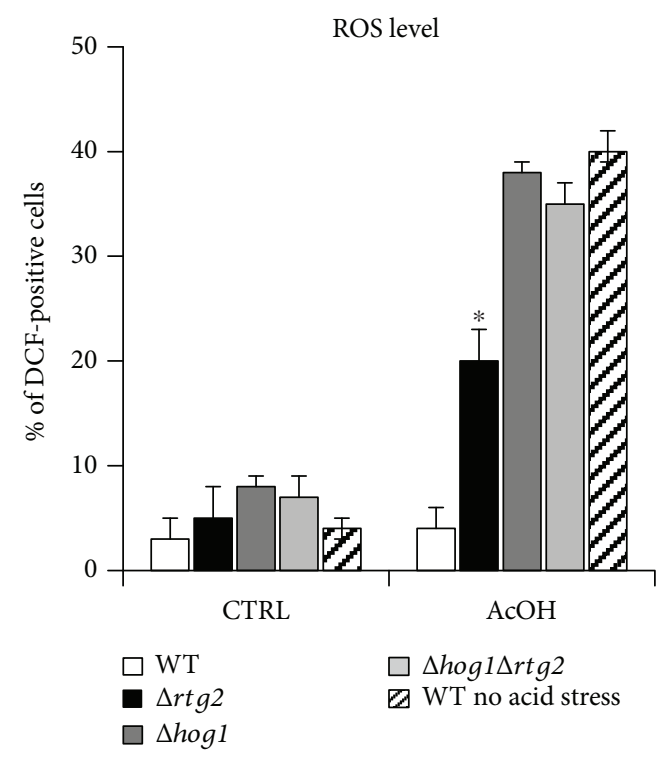

(c)

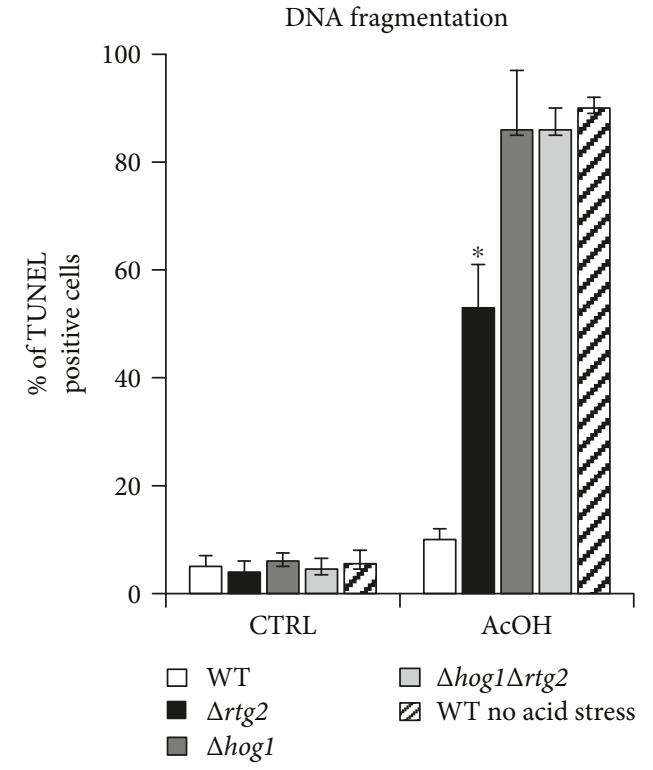

(b)

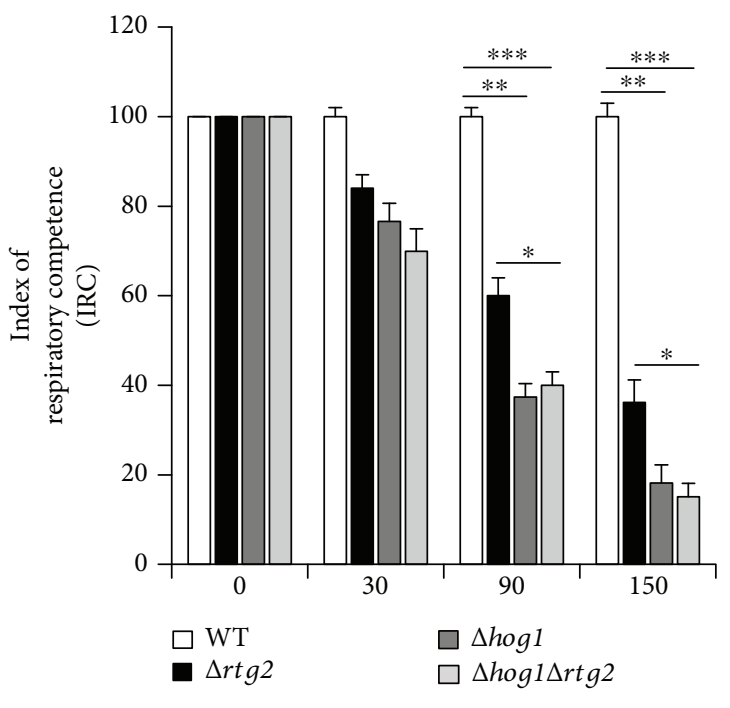

(d)

FIgURe 1: AA-RCD resistance due to acid stress depends on HOG1 and RTG2. (a) Cells were treated with acetic acid (AcOH) after acid stress adaptation, except for WT where indicated. Viability was analyzed at indicated times by measuring colony-forming units (CFU). Cell survival $(100 \%)$ corresponds to the CFU at time zero. Values are shown as the mean \pm standard deviations from four independent experiments. (b, c) DNA fragmentation and intracellular ROS levels were detected by using the TUNEL assay and $\mathrm{H}_{2}$ DCF-DA probe, respectively. The percentage of TUNEL- and DCF-positive cells is reported at $150 \mathrm{~min}$ and $30 \mathrm{~min}$, respectively, with (AcOH) and without (CTRL) acetic acid treatment. In both cases, at least 400 cells were analyzed in three samples from each of the three independent experiments. Paired Student's $t$-test: statistically significantly different with ${ }^{*} p<0.05$ when comparing $\Delta r t g 2$ versus WT, with or without acid stress adaptation, $\Delta h o g 1$, and $\Delta$ hog1rtg2. (d) The index of respiratory competence (IRC) was measured at the indicated times by plating the same cell number on YPD ( $2 \%$ dextrose) and YPG (3\% glycerol). The IRC was calculated from three independent experiments as the percentage of cells able to grow in both YPD and YPG and reported as \% of acid-stressed WT-adapted cells.

ROS and a decrease in catalase activity (see Figures $1(\mathrm{c})$ and 3). Since Hog1 controls the regulation of CTT1 transcription through the transcription factors Msn2/4 [21], we wondered whether RTG2 might have a role in the expression of either CTT1 or CTA1, coding the cytosolic and peroxisomal catalase, respectively. To this aim, CTT1 and CTA1 mRNA levels were measured in WT, $\Delta r \operatorname{tg} 2$, and/or $\Delta$ hog 1 cells either in untreated cells or acid-stressed cells before and after acetic acid addition. We found that the CTT1 transcription profile mirrored catalase activity measured in the same cells and 


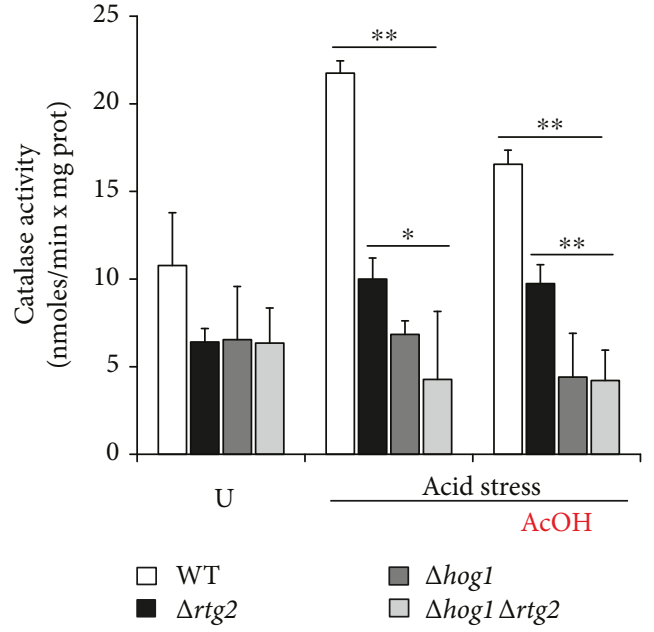

(a)

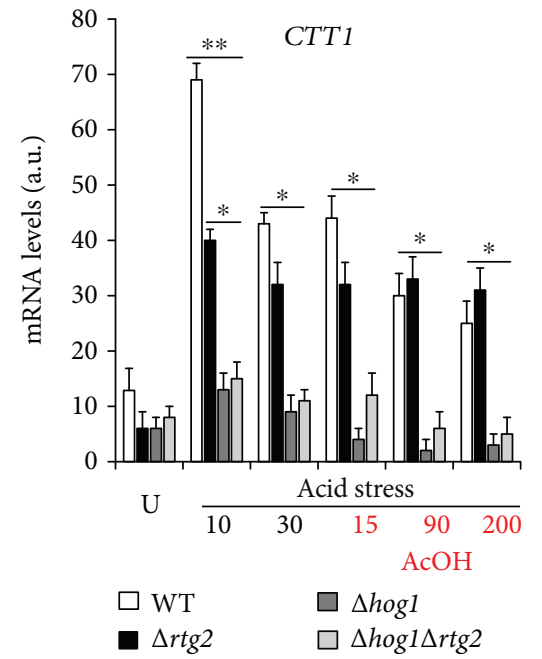

(b)

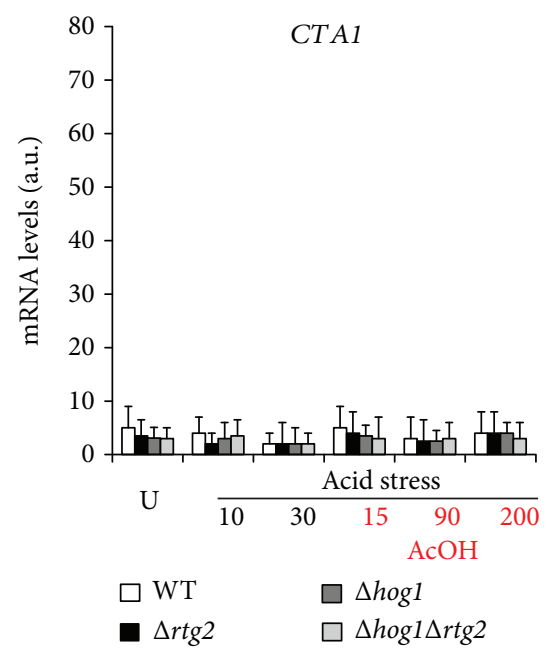

(c)

FIGURE 2: CTT1 mRNA upregulation triggered by acid stress is impaired in $\Delta r t g 2$ mutants. Cells were grown up to exponential phase (U), incubated at low $\mathrm{pH}$ (acid stress), and treated with acetic acid (AcOH) at the indicated times, respectively. (a) Catalase activities were determined on cell extracts at $30 \mathrm{~min}$ of acid stress and after $30 \mathrm{~min}$ of acetic acid exposure as described in Materials and Methods. Values are shown as the mean \pm standard deviations from three independent experiments. Paired Student's $t$-test: statistically significantly different with ${ }^{* *} p<0.005$ when comparing WT versus $\Delta r t g 2, \Delta h o g 1$, and $\Delta$ hog 1 rtg2 and comparing $\Delta$ rtg2 versus $\Delta$ hog1 and $\Delta$ hog1rtg2 at low $\mathrm{pH}$ and with acetic acid and with ${ }^{*} p<0.05$ when comparing $\Delta r \operatorname{tg} 2$ versus $\Delta$ hog1 and $\Delta$ hog1rtg2 at low $\mathrm{pH}$. (b, c) CTT1 and CTA1 mRNA levels were measured by real-time PCR at the indicated times in WT and mutant cells. In both cases, mRNA levels were normalized to that of ACT1 mRNA and reported as means with standard deviation in fluorescence arbitrary units (a.u.). Paired Student's $t$ -test: statistically significantly different with ${ }^{* *} p<0.005$ when comparing WT versus $\Delta r \operatorname{tg} 2, \Delta h \circ g 1$, and $\Delta$ hog1rtg2; with ${ }^{*} p<0.05$ when comparing $\Delta r t g 2$ versus $\Delta$ hog 1 and $\Delta$ hog $1 r t g 2$ at $10 \mathrm{~min}$ low $\mathrm{pH}$; and with ${ }^{*} p<0.05$ when comparing WT and $\Delta r t g 2$ versus $\Delta h o g 1$ and $\Delta$ hoglrtg2 at $30 \mathrm{~min}$ low $\mathrm{pH}$ and with acetic acid.

under the same conditions (Figure 3(b)). Indeed, RTG2 deletion strongly inhibited Hog1-dependenttranscription in untreated and acid stress conditions. The CTT1 mRNA level is rapidly and transiently increased up to 5 -fold after 10 minutes of acid stress (about 70 a.u.) and decreased to about 45 a.u. after $30 \mathrm{~min}$ in WT cells. No changes were observed for CTT1 transcription following acetic acid treatment. On the other hand, CTT1 expression was 40 a.u. after $10 \mathrm{~min}$ of acid stress and remained constant also during the acetic acid treatment in $\Delta r \operatorname{rg} 2$ cells (Figure 2(b)). It is of note that in all conditions tested, no CTA1 transcription was observed in any strains analyzed (Figure 2(c)).

These data showed that only catalase T activity is responsible for AA-RCD evasion in acid-stressed cells. They also confirm that CTT1 mRNA upregulation following acid stress strictly depends on SAPK Hog 1 kinase and gave the first evidence that Rtg2 is required for Hog1-dependent CTT1 mRNA upregulation in response to acid stress.

3.3. RTG2 Deletion Causes a Delay in Hog1 Phosphorylation. Hog1 activation occurs through its phosphorylation and translocation from the cytoplasm to the nucleus, which is required for transcriptional regulation [22]. Thus, to gain insights into how RTG2 may alter Hog1-dependent transcription, we monitored the Hog1 phosphorylation state in acid-stressed WT or RTG2-lacking cells with or without acetic acid treatment (Figure 4). Under unstressed conditions, the phosphorylated protein was almost undetectable in both WT and mutant cells. Hog1 phosphorylation ensued in acid-stressed WT, but not in $\Delta r \operatorname{tg} 2$ cells after 5 and 10 minutes. At 30 minutes, while phosphorylation increased in $\Delta r \operatorname{tg} 2$ cells, a reduction in the amount of phosphorylated protein was observed in WT cells (Figure 3(a)). During acetic acid treatment, Hog1 showed a level of phosphorylation in WT cells virtually similar to that of $\Delta r \operatorname{tg} 2$ cells at 30,60 , and $90 \mathrm{~min}$ (Figure 3(b)).

Thus, RTG2 can modulate the phosphorylation of Hog1 causing a delay in the activation of Hog1 kinase at least in response to acid stress.

3.4. Rtg2 Does Not Activate Rtg1/3-Dependent Retrograde Target Gene Transcription in Acid Stressed Cells. We have previously shown that yeast cells grown in raffinose, which differently from glucose, promotes respiration, evade AA-RCD due to RTG pathway activation $[15,23]$. In order to check if RTG pathway activation can contribute to AA-RCD evasion of acid-stressed cells, the mRNA level of CIT2, encoding the peroxisomal isoform of citrate synthase, whose upregulation is a hallmark of Rtg1/3-dependent transcription activation [14], was measured in WT and $\Delta h o g 1$ mutant cells either in normal growth conditions without acetic acid (untreated) or during acid stress before and after acetic acid addition (Figure 4). The level of expression was about 5 a.u. in the absence of stress. Interestingly, about a 2 -fold reduction of the CIT2 mRNA level of expression was measured in $\Delta$ hog1 cells under normal growth conditions. The CIT2 mRNA level showed about an $80 \%$ decrease in acid-stressed WT cells with respect to normal growth 

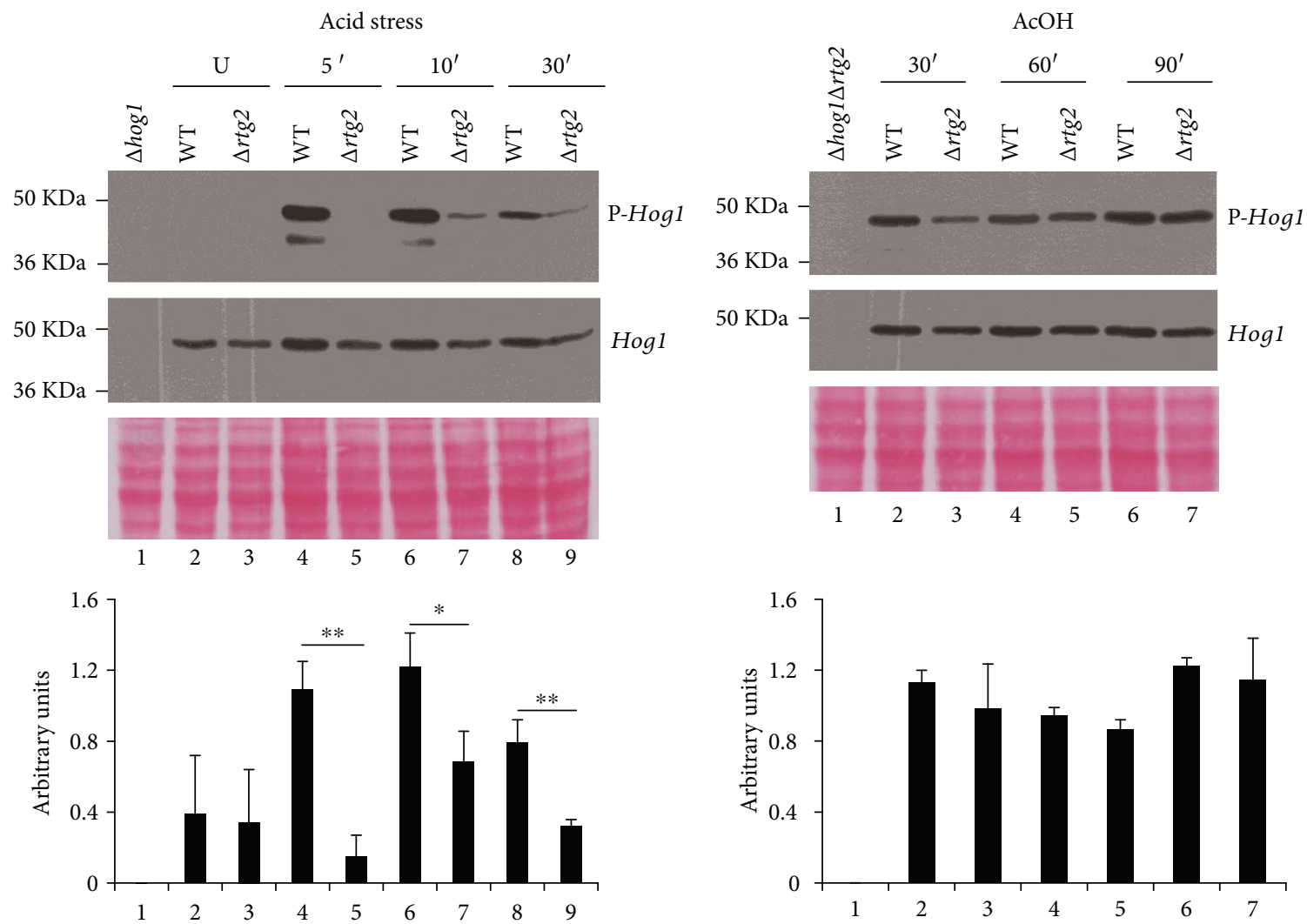

(a)

(b)

FIGURE 3: Hog1 phosphorylation in WT and $\Delta r t g 2$ mutants. (a) Cells were grown up to exponential phase (U), incubated at low pH, and (b) treated with acetic acid $(\mathrm{AcOH})$. Cell protein extracts were prepared from WT and $\Delta$ rtg2 cells at the indicated times and analyzed by immunoblot with anti-P-Hoglp and anti-Hog1p antibodies. Cell extracts from $\Delta$ hog1 and $\Delta$ hog $1 \Delta$ rtg2 cells have been analyzed as a negative control. Ponceau staining was used as loading control. The histogram reports the ratio between phosphorylated (P-Hog1) and total Hogl as quantified by densitometric analysis of the film normalized with Ponceau staining and expressed in arbitrary units. Reported values are the means \pm standard deviations of three independent experiments. Paired Student's $t$-test: statistically significantly different with ${ }^{* *} p<0.05$ and ${ }^{*} p<0.1$ when comparing $\Delta r \operatorname{rg} 2$ versus WT under acid stress.

conditions and exhibited a virtually constant value either in the absence or in the presence of acetic acid. This shows that the RTG pathway was not activated by acid stress. On the other hand, the CIT2 mRNA level remained unchanged as compared with normal growth conditions in acid-stressed $\Delta$ hogl cells with or without acetic acid treatment (Figure 5). As expected, CIT2 expression was completely abolished in the $\Delta r t g 2$ mutant.

These data demonstrate that the RTG signaling does not contribute to AA-RCD evasion in cells adapted by acid stress. In addition, Hog 1 is required for the full transcriptional activation of CIT2 in normal growth conditions as well as for the CIT2 transcription regulation in response to stress.

\section{Discussion}

Yeast cells can evade AA-RCD either upon acid stress adaptation, due to the cytoprotective role of the catalase upregulation, or under conditions of concomitant derepression of mitochondrial respiration and RTG pathway activation, like in raffinose-grown cells $[6,8,15]$. In this work, we showed a mutual interplay between $R T G 2$, a positive regulator of the mitochondrial RTG pathway, and SAPK HOG1, the master regulator of catalase gene transcription, in coordinating stress response and modulating resistance to AA-RCD due to acid stress adaptation.

Our data demonstrate that both HOG1 and RTG2 contribute to AA-RCD evasion, with an essential requirement of HOG1 (Figures 1(a) and 2(b)). These two genes seem to exert their protective role against acetic acid toxicity by increasing oxidative stress resistance and preserving mitochondrial function (Figures 1(c) and 1(d)). The requirement of RTG2 for CTT1 activation, as shown in Figure 3(a), supports the involvement of the RTG pathway in oxidative stress resistance as already shown in [13, 24, 25]. Complete preservation of mitochondrial function in acid stress cells evading AA-RCD was abolished by HOG1 deletion, confirming the link of the MAPK pathway with mitochondrial dysfunction already observed upon osmotic stress and in pathogenic fungi $[24,26,27]$.

Here, we showed that specific activation of cytosolic catalase $\mathrm{T}$, but not peroxisomal/mitochondrial catalase $\mathrm{A}$, is responsible for acid stress cell evasion of AA-RCD (Figure 2). This is in agreement with previous findings that CTT1 overexpression can fully prevent AA-RCD [8]. Thus, detoxification of hydrogen peroxide together with catalase 


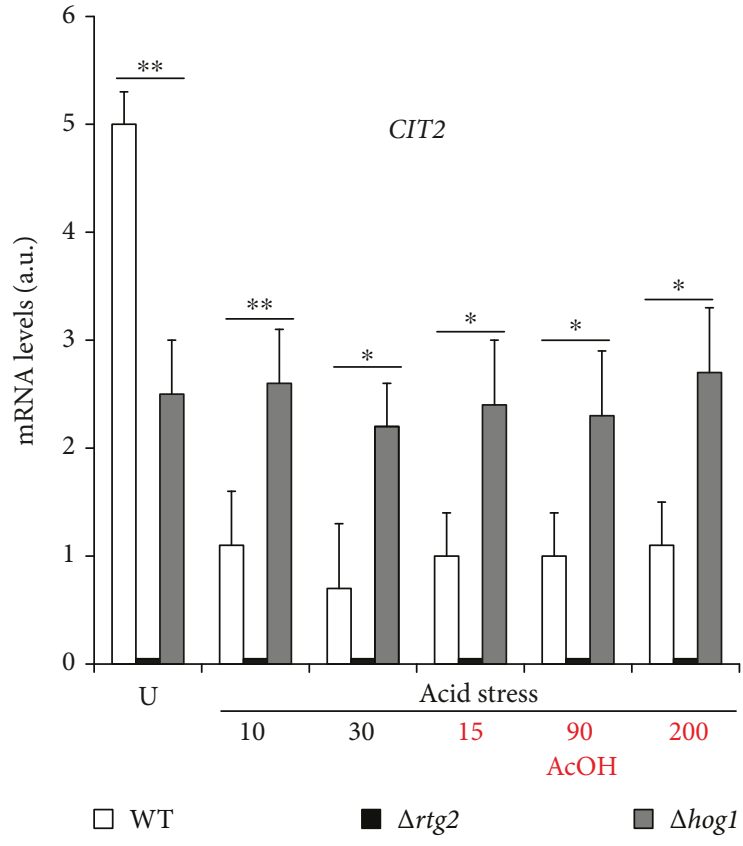

FIGURE 4: CIT2 expression in $\Delta$ hog1 mutants. Cells were grown up to exponential phase $(\mathrm{U})$, incubated at low $\mathrm{pH}$, and treated with acetic acid $(\mathrm{AcOH})$. CIT2 mRNA levels were measured by real-time PCR at the indicated times in WT and mutant cells from three independent experiments. CIT2 mRNA levels were normalized to that of ACT1 mRNA and reported as means with standard deviation in fluorescence arbitrary units (a.u.). Paired Student's $t$-test: statistically significantly different with ${ }^{* *} p<0.1$ when comparing WT versus $\Delta r t g 2$ and $\Delta h o g 1$ in untreated cells and at $10 \mathrm{~min}$ low $\mathrm{pH}$ and with ${ }^{*} p<0.5$ when comparing WT versus $\Delta h o g 1$ and $\Delta r \operatorname{tg} 2$ at $30 \mathrm{~min}$ low $\mathrm{pH}$ and with acetic acid.

activation appears to be a key condition for acetic acid tolerance and in general stress cotolerance as reported in $[28]$.

How RTG2 interacts with HOG1 to regulate CTT1 transcription remains to be established. Our analysis of Hog1 phosphorylation en route to acid stress clearly shows that RTG2 deletion significantly delays Hog1 activation through phosphorylation, suggesting possible interactions with Hog1-specific kinases (Figure 3). On the other hand, HOG1 has been involved in the regulation of Rtg1/3 complex activity in response to osmostress [16]. According to these results, our data showed that HOG1 could exert a modulatory effect on Rtg1/3-dependent transcription depending on the metabolic state of the cell: Hog1 is required for the full transcriptional activation of the CIT2 gene in normally growing cells, but it negatively regulates RTG pathway activation in stressed cells (Figure 4). This is in agreement with the increase of CIT2 expression occurring in a Hog1-dependent manner upon osmostress [16]. Overall, our data clearly demonstrate the interplay between Rtg2 and Hog1 (Figure 5), whose mechanism deserves further investigations. However, since Rtg2 can bind to chromatin at the promoters of regulated genes as a component of a SAGA-like complex, named SLIK [29], and an interaction between Hogl SAPK and SAGA complex has been previously reported upon severe osmostress [22, 30-32], we can hypothesize that Rtg2 and

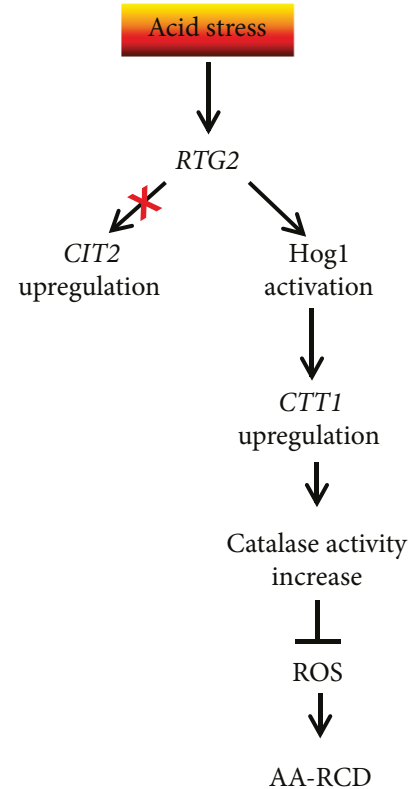

FIgURe 5: A model for the AA-RCD adaptive pathway due to acid stress. Acid stress triggers resistance to AA-RCD through a pathway in which RTG2 does not activate the CIT2 upregulation, i.e., mitochondrial retrograde response, but it is required for $\operatorname{Hog} 1$ activation which controls the transcriptional upregulation of CTT1, encoding cytosolic catalase. The concomitant increase of catalase activity inhibits the ROS-dependent pathway causing AA-RCD.

Hog1 interact at the level of epigenetic regulation of gene expression. In any case, our results clearly show a strict cooperation of HOG and RTG pathways to induce a hormetic response of yeast cells to different kinds of stresses.

It is worth noting that HOG pathway activation can rescue cells from various cellular dysfunctions caused by the impaired biosynthesis of complex sphingolipids through a yet unknown molecular mechanism [33]. It has been suggested that the HOG pathway could sense impaired formation of lipid microdomains, which are formed by complex sphingolipids and sterol molecules [34]. Since others and we have shown that ceramide production contributes to mitochondrial dysfunction and AA-RCD $[35,36]$, our results support the hypothesis (it is tempting to speculate) that acid stress could activate Hog1 through a mechanism alike that ensues a sphingolipid metabolism defect.

Differently from raffinose-grown cells which evade AARCD mostly due to RTG pathway activation [15], acid stress does not activate Rtg1/3-dependent mitochondrial retrograde target gene transcription for cell adaptation (Figure 4). Thus, different branches of the RTG-dependent signaling can be activated depending on the type of external stimuli, suggesting that $R T G 2$ may have a complex role in the integration of different stress signaling pathways through yet unknown regulatory elements [37].

\section{Conclusions}

By further exploring the molecular basis of yeast tolerance to acetic acid, this work highlights the importance of detailed 
understanding of the interplay between signaling pathways in stress response. In particular, it provides novel insights into the strict relationship between cell stress adaptation and oxidative stress resistance ensuing from the cooperation of RTG- and HOG1-mediated stress signaling pathways. When activated, these pathways converge to produce a hormetic response in cells undergoing environmental stresses. The discovery of the new role for RTG2 in modulating Hog1 activation will deserve deeper investigations to understand conditions and modes of this interaction upon stress. These results could be relevant for certain industrial microbial applications in which acetic acid accumulation is detrimental and the improvement of strain resistance to acetic acid is highly desirable.

\section{Data Availability}

The data used to support the findings of this study are available from the corresponding author upon request.

\section{Conflicts of Interest}

The authors declare that there is no conflict of interest regarding the publication of this paper.

\section{Acknowledgments}

The authors thank Dr. Laura Marra for her administrative and technical assistance and are grateful to Dr. Ersilia Marra for her support and critical advices in starting this project. This work was supported by Progetto POR Puglia FESR-FSE 2014-2020 Azione 1.6. Avviso "Innonetwork" (grant number 1JLZKD0).

\section{References}

[1] L. Portt, G. Norman, C. Clapp, M. Greenwood, and M. T. Greenwood, "Anti-apoptosis and cell survival: a review," Biochimica et Biophysica Acta (BBA) - Molecular Cell Research, vol. 1813, no. 1, pp. 238-259, 2011.

[2] M. Zdralević, N. Guaragnella, L. Antonacci, E. Marra, and S. Giannattasio, "Yeast as a tool to study signaling pathways in mitochondrial stress response and cytoprotection," Scientific World Journal, vol. 2012, article 912147, 10 pages, 2012.

[3] A. P. Gasch, P. T. Spellman, C. M. Kao et al., "Genomic expression programs in the response of yeast cells to environmental changes," Molecular Biology of the Cell, vol. 11, no. 12, pp. 4241-4257, 2000.

[4] D. Carmona-Gutierrez, M. A. Bauer, A. Zimmermann et al., "Guidelines and recommendations on yeast cell death nomenclature," Microbial Cell, vol. 5, no. 1, pp. 4-31, 2018.

[5] N. Guaragnella, L. Antonacci, S. Passarella, E. Marra, and S. Giannattasio, "Achievements and perspectives in yeast acetic acid-induced programmed cell death pathways," Biochemical Society Transactions, vol. 39, no. 5, pp. 1538-1543, 2011.

[6] S. Giannattasio, N. Guaragnella, M. Corte-Real, S. Passarella, and E. Marra, "Acid stress adaptation protects Saccharomyces cerevisiae from acetic acid-induced programmed cell death," Gene, vol. 354, pp. 93-98, 2005.
[7] N. Guaragnella, L. Antonacci, S. Passarella, E. Marra, and S. Giannattasio, "Hydrogen peroxide and superoxide anion production during acetic acid-induced yeast programmed cell death," Folia Microbiologia (Praha), vol. 52, no. 3, pp. 237240, 2007.

[8] N. Guaragnella, L. Antonacci, S. Giannattasio, E. Marra, and S. Passarella, "Catalase $\mathrm{T}$ and $\mathrm{Cu}, \mathrm{Zn}$-superoxide dismutase in the acetic acid-induced programmed cell death inSaccharomyces cerevisiae," FEBS Letters, vol. 582, no. 2, pp. 210-214, 2008.

[9] R. Wieser, G. Adam, A. Wagner et al., "Heat shock factorindependent heat control of transcription of the CTT1 gene encoding the cytosolic catalase T of Saccharomyces cerevisiae," The Journal of Biological Chemistry, vol. 266, no. 19, pp. 12406-12411, 1991.

[10] C. Schüller, J. L. Brewster, M. R. Alexander, M. C. Gustin, and H. Ruis, "The HOG pathway controls osmotic regulation of transcription via the stress response element (STRE) of the Saccharomyces cerevisiae CTT1 gene," The EMBO Journal, vol. 13, no. 18, pp. 4382-4389, 1994.

[11] J. L. Brewster and M. C. Gustin, "Hog1: 20 years of discovery and impact," Science Signaling, vol. 7, no. 343, p. re7, 2014.

[12] M. Mollapour and P. W. Piper, "Hog1p mitogen-activated protein kinase determines acetic acid resistance in Saccharomyces cerevisiae," FEMS Yeast Research, vol. 6, no. 8, pp. 1274-1280, 2006.

[13] M. Mollapour and P. W. Piper, "Hog1 mitogen-activated protein kinase phosphorylation targets the yeast Fps1 aquaglyceroporin for endocytosis, thereby rendering cells resistant to acetic acid," Molecular and Cellular Biology, vol. 27, no. 18, pp. 6446-6456, 2007.

[14] Z. Liu and R. A. Butow, "Mitochondrial retrograde signaling," Annual Review of Genetics, vol. 40, no. 1, pp. 159-185, 2006.

[15] N. Guaragnella, M. Ždralević, P. Lattanzio et al., "Yeast growth in raffinose results in resistance to acetic-acid induced programmed cell death mostly due to the activation of the mitochondrial retrograde pathway," Biochimica et Biophysica Acta (BBA) - Molecular Cell Research, vol. 1833, no. 12, pp. 2765-2774, 2013.

[16] C. Ruiz-Roig, N. Noriega, A. Duch, F. Posas, and E. de Nadal, "The Hog1 SAPK controls the Rtg1/Rtg3 transcriptional complex activity by multiple regulatory mechanisms," Molecular Biology of the Cell, vol. 23, no. 21, pp. 4286-4296, 2012.

[17] E. Parrella and V. D. Longo, "The chronological life span of Saccharomyces cerevisiae to study mitochondrial dysfunction and disease," Methods, vol. 46, no. 4, pp. 256-262, 2008.

[18] H. Luck, "Quantitative determination of catalase activity of biological material," Enzymologia, vol. 17, no. 1, pp. 31-40, 1954.

[19] R. Ossig, C. Dascher, H. H. Trepte, H. D. Schmitt, and D. Gallwitz, "The yeast SLY gene products, suppressors of defects in the essential GTP-binding Ypt1 protein, may act in endoplasmic reticulum-to-Golgi transport," Molecular and Cellular Biology, vol. 11, no. 6, pp. 2980-2993, 1991.

[20] S. Giannattasio, A. Atlante, L. Antonacci et al., "Cytochrome c is released from coupled mitochondria of yeast en route to acetic acid-induced programmed cell death and can work as an electron donor and a ROS scavenger," FEBS Letters, vol. 582, no. 10, pp. 1519-1525, 2008.

[21] M. T. Martínez-Pastor, G. Marchler, C. Schüller, A. MarchlerBauer, H. Ruis, and F. Estruch, "The Saccharomyces cerevisiae 
zinc finger proteins Msn2p and Msn4p are required for transcriptional induction through the stress response element (STRE)," The EMBO Journal, vol. 15, no. 9, pp. 2227-2235, 1996.

[22] Vladimír Reiser, H. Ruis, and G. Ammerer, "Kinase activitydependent nuclear export opposes stress- induced nuclear accumulation and retention of Hog1 mitogen-activated protein kinase in the budding yeast Saccharomyces cerevisiae," Molecular Biology of the Cell, vol. 10, no. 4, pp. 1147-1161, 1999.

[23] L. Laera, N. Guaragnella, M. Ždralević, D. Marzulli, Z. Liu, and S. Giannattasio, "The transcription factors ADR1 or CAT8 are required for RTG pathway activation and evasion from yeast acetic acid-induced programmed cell death in raffinose," Microbial Cell, vol. 3, no. 12, pp. 621-631, 2016.

[24] N. Q. Torelli, J. R. Ferreira-Júnior, A. J. Kowaltowski, and F. M. da Cunha, "RTG1- and RTG2-dependent retrograde signaling controls mitochondrial activity and stress resistance in Saccharomyces cerevisiae," Free Radical Biology \& Medicine, vol. 81, pp. 30-37, 2015.

[25] R. M. N. Friis, J. P. Glaves, T. Huan, L. Li, B. D. Sykes, and M. C. Schultz, "Rewiring AMPK and mitochondrial retrograde signaling for metabolic control of aging and histone acetylation in respiratory-defective cells," Cell Reports, vol. 7, no. 2, pp. 565-574, 2014.

[26] M. M. Pastor, M. Proft, and A. Pascual-Ahuir, "Mitochondrial function is an inducible determinant of osmotic stress adaptation in yeast," The Journal of Biological Chemistry, vol. 284, no. 44, pp. 30307-30317, 2009.

[27] R. Alonso-Monge, S. Carvaihlo, C. Nombela, E. Rial, and J. Pla, "The Hog1 MAP kinase controls respiratory metabolism in the fungal pathogen Candida albicans," Microbiology, vol. 155, no. 2, pp. 413-423, 2009.

[28] N. Gurdo, G. F. Novelli Poisson, Á. B. Juárez, M. C. Rios de Molina, and M. A. Galvagno, "Improved robustness of an ethanologenic yeast strain through adaptive evolution in acetic acid is associated with its enzymatic antioxidant ability," Journal of Applied Microbiology, vol. 125, no. 3, pp. 766-776, 2018.

[29] M. G. Pray-Grant, D. Schieltz, S. J. McMahon et al., “The novel SLIK histone acetyltransferase complex functions in the yeast retrograde response pathway," Molecular and Cellular Biology, vol. 22, no. 24, pp. 8774-8786, 2002.

[30] M. Proft and K. Struhl, "Hog1 kinase converts the Sko1-Cyc8-Tup1 repressor complex into an activator that recruits SAGA and SWI/SNF in response to osmotic stress," Molecular Cell, vol. 9, no. 6, pp. 1307-1317, 2002.

[31] M. Zapater, M. Sohrmann, M. Peter, F. Posas, and E. de Nadal, "Selective requirement for SAGA in Hog1-mediated gene expression depending on the severity of the external osmostress conditions," Molecular and Cellular Biology, vol. 27, no. 11, pp. 3900-3910, 2007.

[32] S. Hohmann, "Osmotic stress signaling and osmoadaptation in yeasts," Microbiology and Molecular Biology Reviews, vol. 66, no. 2, pp. 300-372, 2002.

[33] Y. Yamaguchi, Y. Katsuki, S. Tanaka et al., "Protective role of the HOG pathway against the growth defect caused by impaired biosynthesis of complex sphingolipids in yeast Saccharomyces cerevisiae," Molecular Microbiology, vol. 107, no. 3, pp. 363-386, 2018.

[34] M. Tani and K. Funato, "Protection mechanisms against aberrant metabolism of sphingolipids in budding yeast," Current Genetics, vol. 64, no. 5, pp. 1021-1028, 2018.
[35] A. Rego, M. Costa, S. R. Chaves et al., "Modulation of mitochondrial outer membrane permeabilization and apoptosis by ceramide metabolism," PLoS One, vol. 7, no. 11, article e48571, 2012.

[36] V. Longo, M. Ždralević, N. Guaragnella, S. Giannattasio, L. Zolla, and A. M. Timperio, "Proteome and metabolome profiling of wild-type and YCA1-knock-out yeast cells during acetic acid-induced programmed cell death," Journal of Proteomics, vol. 128, pp. 173-188, 2015.

[37] K. Podholová, V. Plocek, S. Rešetárová et al., “Divergent branches of mitochondrial signaling regulate specific genes and the viability of specialized cell types of differentiated yeast colonies," Oncotarget, vol. 7, no. 13, pp. 15299-15314, 2016. 


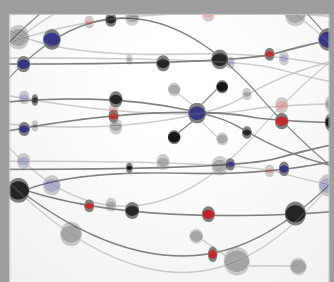

The Scientific World Journal
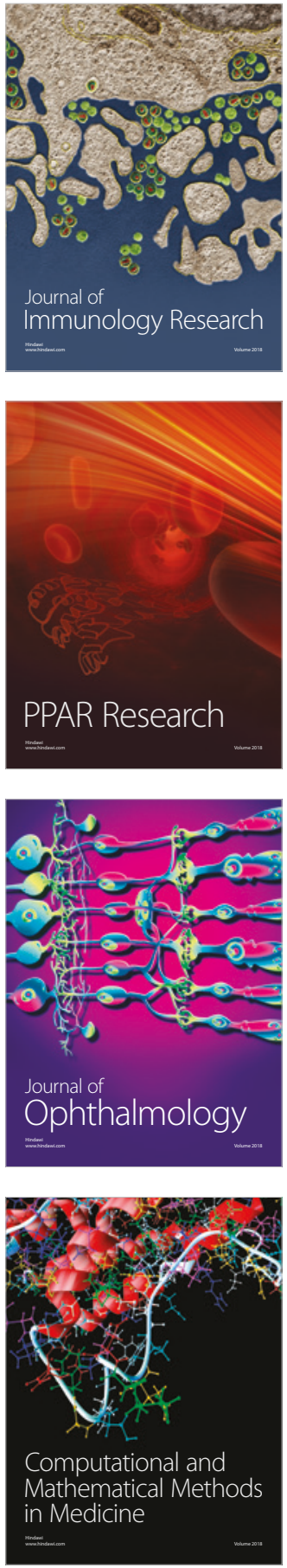

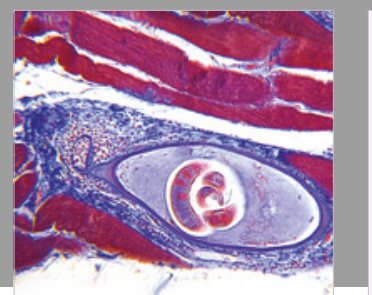

Gastroenterology Research and Practice

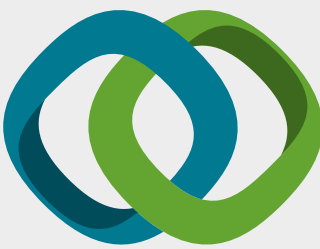

\section{Hindawi}

Submit your manuscripts at

www.hindawi.com
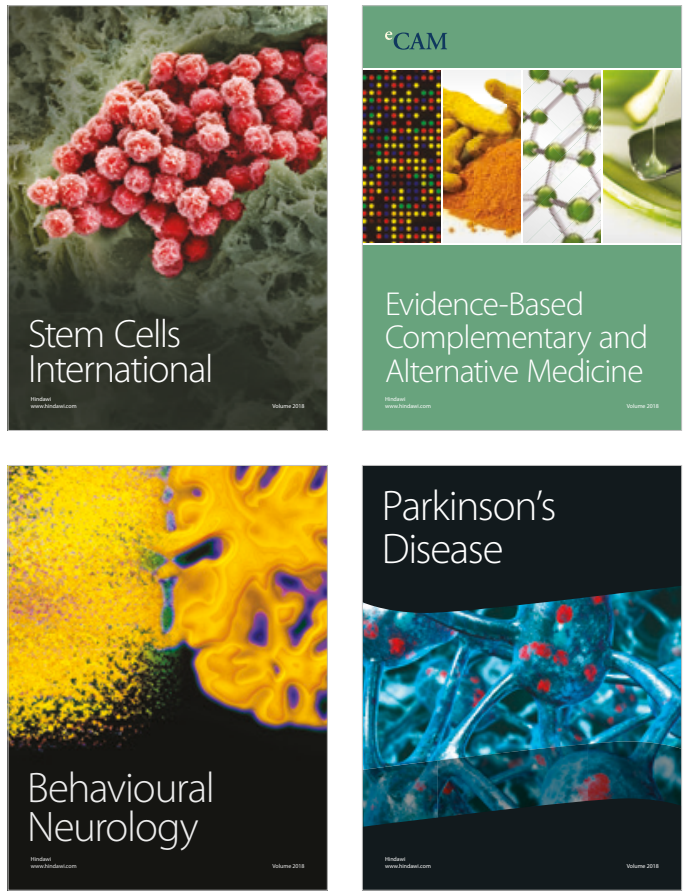

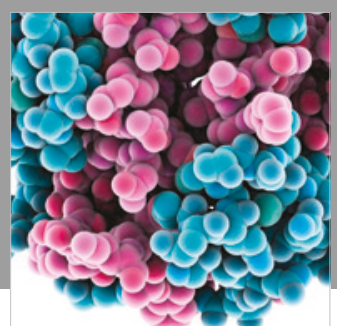

ournal of

Diabetes Research

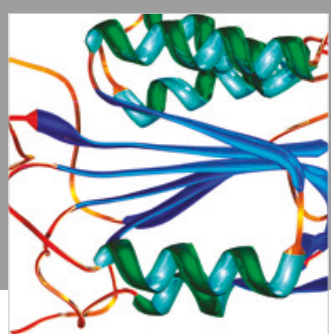

Disease Markers
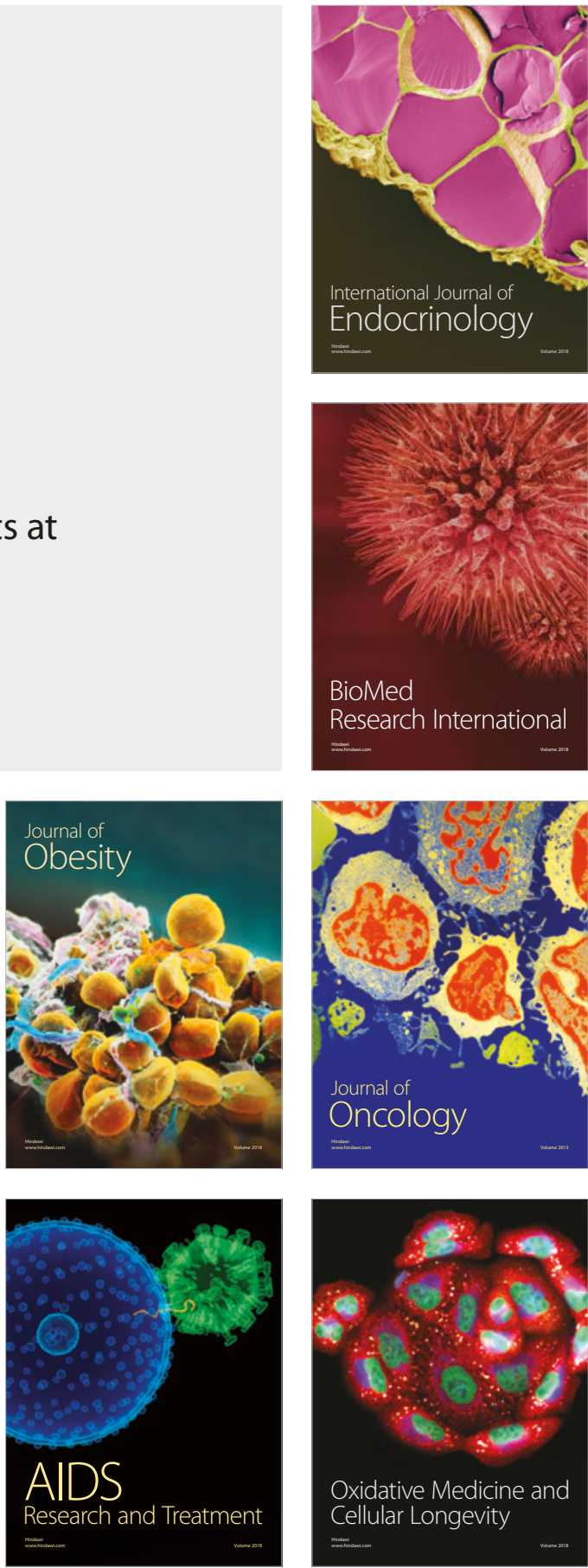